\title{
Efficacy and Safety of a Novel Mixture for Submucosal Injection During Endoscopic Mucosal Resection
}

\author{
Thomas Hallgren* and Thomas Andersson Thell \\ Department of Surgery, Central Hospital Karlstad, Sweden \\ *Corresponding author: Thomas Hallgren, Department of Surgery, Central Hospital, S-652 30 Karlstad, Sweden
}

\section{ARTICLE INFO}

Received: 醳 January 20, 2021

Published: 仹 January 26, 2021

Citation: Thomas Hallgren, Thomas Andersson Thell. Efficacy and Safety of a Novel Mixture for Submucosal Injection During Endoscopic Mucosal Resection. Biomed J Sci \& Tech Res 33(3)-2021. BJSTR. MS.ID.005393.

\section{ABSTRACT}

Introduction: Colorectal polyps may develop into cancer and should therefore be removed. For larger polyps ( $>10 \mathrm{~mm})$ this is preferably done by endoscopic mucosal resection. To minimize complications larger polyps should be lifted by a submucosal injection of a liquid before polypectomy. The present study aims to investigate the feasibility of a newly developed liquid for this purpose.

Methods: Routine colonoscopy patients with polyps needing removal were included in the study. Submucosal injection was given before the resection of polyps between 10 to $30 \mathrm{~mm}$ in diameter and the effects and complications were followed, and userfriendliness of the liquid and packaging was judged.

Results: 48 polypectomies in 37 patients were performed. In all patients a good lift was noted throughout the procedure and no reinjection was needed. No complications were noted. The user-friendliness was rated as very good in $83 \%$.

Conclusion: The tested mixture very well meets the set requirements for a safe and radical removal of colonic polyps in size up to $30 \times 30 \mathrm{~mm}$. The packaging and liquid have a high degree of user-friendliness.

\section{Introduction}

Colorectal cancer (CRC) is one of the leading causes of cancerrelated death worldwide [1], and adenomatous polyps in the colon and rectum are well-known precursor lesions for this malignancy [2]. It is therefore of vital importance to detect and radically remove such polyps to reduce the incidence as well as the mortality of CRC [3]. Colonoscopy is the optimal modality for the detection and simultaneous removal of colorectal polyps, one of the most frequent findings during colonoscopy. It is, however, often difficult to macroscopically differentiate whether they are of adenomatous character, serrated, or just benign hyperplastic polyps, and it is therefore generally recommended that all polyps found at colonoscopy should be removed [4]. Submucosal injection of a liquid is generally required before endoscopic mucosal resection (EMR), the recommended method for removal of polyps in the colon larger than $10 \mathrm{~mm}$ according to guidelines from the European Society of Gastrointestinal Endoscopy (ESGE) and others [4].

The injection is done to minimize the risks for perforation as well as postpolypectomy bleeding, the two most frequent and feared complications to this procedure [5]. Even though saline has been the most frequent liquid used, several other different solutions have been tried, all with different types of drawbacks, the most common being rapid diffusion into surrounding tissue, and thus losing the desired "lift effect". In the present study a newly developed premixed solution for submucosal injection prior to endoscopic polypectomy was evaluated. The study was designed as a feasibility study and aimed to evaluate the efficacy, safety as well as user-friendliness of this novel mixture and its packaging.

\section{Methods and Material}

The study liquid, "Pollift", is a newly developed premixed colorless solution consisting of glycerol and hypertonic saline filled into pressurized and sterilized $100 \mathrm{ml}$ bottles. The solution in the bottle is sterile for three years, even after start of use. Inclusion criteria were patients planned for routine colonoscopy for a positive fecal blood test or diffuse bowel symptoms at the endoscopy unit at Central Hospital, Karlstad. The patients were informed of the study prior to the colonoscopy, verbally as well as in writing, and gave a 
written consent. The examinations were all performed by one of three very experienced fulltime endoscopists. Colorectal polyps with a size between $10 \times 10 \mathrm{~mm}$ up to $30 \times 30 \mathrm{~mm}$, found during colonoscopy were considered for inclusion. Exclusion criteria were age below 18 years, incapacity to understand the study information and polyps with a malignant endoscopic appearance. If a polyp within the actual size range, and needing removal, was detected, and the patient prior had accepted participation in the study, the polypectomy procedure was included.

When a polyp was detected, it was cleaned and thereafter inspected with withe light and NBI (Narrow Band Imaging). Injection was performed with 1- $3 \mathrm{ml}$ of the studied liquid in 1-3 locations around/under the polyp depending on size and location. After injection, the polypectomy was performed using a hot snare. All pieces of the polyps were retrieved for histopathological examination. Photo documentation was done before as well as after the procedure. All patients were observed after the procedure for at least sixty minutes and any sign of adverse events were registered. Fourteen days after the procedure the patients were interviewed via telephone for any symptoms suggestive of a complication that might be associated with the procedure. The assisting personnel were interviewed after each procedure regarding handling and user-friendliness of the packaging. Functionality was judged on according to an arbitrary scale from 1 to 10 , where 1 is considered as "very bad" and 10 as "very good".

\section{Ethics}

The study protocol was approved by the Ethical Committee of Uppsala University, registration number 2018/047.

\section{Results}

37 patients with a mean age of 67 years (range of 36 to 84 ) were included in the study, 20 (54\%) were female and 17 (46\%) were male. In these patients 48 polypectomies were performed, one only in 32, two in 5 and three in 2 patients. The majority of polyps were localized in the proximal part of the colon (caecum to right flexure), nine caecum, sixteen right colon and six in the right flexure. Five polyps were located in the transverse colon, and from the left flexure and down the sigmoid seven polyps were removed and finally five in the rectum. Polyp size varied from 10x10 mm to $25 \times 25 \mathrm{~mm}$. The mean total volume of fluid used for submucosal injection was 3,3 $\mathrm{ml}$ (range 1-7 ml), and no reinjection was needed. The mean time for resection of a single polyp was 4,6 min (range 2-15). The lift duration from the injected submucosal fluid was sufficient to perform a complete polypectomy in all patients, even when the procedure lasted up to $15 \mathrm{~min}$, All polyps were macroscopically radically removed, $37(77 \%)$ in one piece, $8(17 \%)$ in two pieces and $3(6 \%)$ in three or more pieces.

Histologically the polyps consisted of 23 (49\%) hyperplastic and serrated non-dysplastic lesions, 19 (40\%) tubular/tubulovillous adenomas with low-grade dysplasia and 5 (11\%) tubular/ tubulovillous adenomas with high grade dysplasia. One polyp was reported as an adenocarcinoma. Not any of the reports indicated tissue damage of the specimen. No complications were recorded during follow-up, neither during observation in the endoscopy unit nor when interviewed two weeks later. Regrading functionality the assisting personnel in $83 \%$ of the procedures the rating was 10. In $6 \%$ the rating was 9 and $12 \%$ the rating was 8 . In the cases where functionality was rated as 9 or 8 a $5 \mathrm{ml}$ syringe was used for injection with a less precise fit to the bottle nipple. After changing to a $10 \mathrm{ml}$ syringe and changing bottle nipple between each patient all procedures were rated as 10 .

\section{Discussion}

The present study is aimed to evaluate a new liquid for submucosal lifting when performing endoscopic resection of colorectal polyps or neoplasms, a technique first published in 1955 concerning rigid sigmoidoscopy and later in 1973 by Deyhle using a flexible colonoscope [6]. The technique has evolved considerably over the years and new instruments, devices and skills have been added. Indications for endoscopic surgery has expanded and success rates improved. Modern screening programmes for colorectal cancer have already, and will over time, significantly increase the number of colonoscopies performed and thus polyps diagnosed. The neoplasms may, in principle, be of two kinds, flat or stalked, and their size may vary from millimetres to several centimetres, but the majority, 80 to $90 \%$, are less than $10 \mathrm{~mm}$ in size [5]. Stalked neoplasms can be removed more easily by cutting of the stalk by means of a snare. The non-stalked adenomas larger than $10 \mathrm{~mm}$ in diameter, however, need to be separated from the underlying mucosa before removal and this is done by lifting [5].

The lift is achieved by endoscopic injection of a fluid beneath the neoplasm, to enable removal in a safe and complete way. The injection of the lifting fluid also serves as a protection for the underlying muscular layer. The bleb created by the formation of a cushion beneath the neoplasm separates it from the muscular layer, and the resulting demarcation makes it easier to remove the neoplasm without damaging the surrounding tissue. The lifting fluids are developed with the aim of creating a lift that stands long enough to allow a safe and complete polyp removal and without causing unwanted side-effects. Traditionally, various saline solutions have been used creating blebs of a rather short duration and not seldom a reinjection is necessary to complete the procedure [7]. Therefore, other constituents have been tried such as gels, glycerol or sugars such as dextrose or fructose. Dextrose is said to provide a longer lasting bleb than normal saline, but with the drawback of specimen tissue damage.

This effect is said to occur from $20 \%$ upwards [8]. Uraoka considers this effect to seriously question the suitability of dextrose in lifting solutions [9]. Another problem reported is smoke formation. Hyaluronic acid is used to create a long-standing bleb, but this carries a considerably higher cost, and a high viscosity may 
lead to difficulties in injection [10]. The present study has shown that this premixed solution of glycerol and hypertonic saline "Pollift" gives a sufficient lift of polyps up to $30 \mathrm{~mm}$, allowing complete endoscopic resection without any need for reinjection, and seems, so far, not to cause any unwanted side-effects. Furthermore, the cost is relatively low, particularly since the contents in the bottle is kept sterile for up to three years and can be used for multiple procedures during this time. It is also shown to be user-friendly for the assisting personnel.

\section{References}

1 Karuppannan S, Kelty E, Sodhi Berry N (2020) Trends in incidence, mortality rates, and survival of colorectal cancer in Western Australia from 1990 to 2014: a retrospective whole-population longitudinal study. Int J Colorectal Dis 35: 1719-1727.

2 Day DW, Morson BC (1978) The adenoma-carcinoma sequence. Major Probl Pathol 10: 58-71.

3 Song M, Emilsson L, Bozorg SR, Andrew T Chan, Jonas F Ludvigsson, et al. (2020) Risk of colorectal cancer incidence and mortality after polypectomy: a Swedish record-linkage study. Lancet Gastroenterol Hepatol 5(6): 537-547.

ISSN: 2574-1241

DOI: 10.26717/BJSTR.2021.33.005393

Thomas Hallgren. Biomed J Sci \& Tech Res

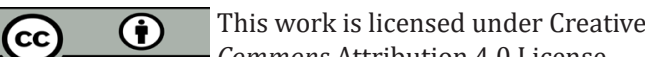

Submission Link: https://biomedres.us/submit-manuscript.php
4 Ferlitsch M, Moss A, Hassan C, Bhandari P (2017) Colorectal polypectomy and endoscopic mucosal resection (EMR): European Society of Gastrointestinal Endoscopy (ESGE) Clinical Guideline. Endoscopy 49: 270-297.

5 Ma MX, Bourke MJ (2016) Complications of endoscopic polypectomy, endoscopic mucosal resection and endoscopic submucosal dissection in the colon. Best Pract Res Clin Gastroenterol 30(5): 749-767.

6 Deyhle P, Jenny S, Fumagalli I (1973) [Endoscopic polypectomy in the proximal colon. A diagnostic, therapeutic (and preventive?) intervention]. Dtsch Med Wochenschr 98(5): 219-220.

7 Norton ID, Wang L, Levine SA, Christopher J Gostout, Bret T Petersen, et al. (2002) Efficacy of colonic submucosal saline solution injection for the reduction of iatrogenic thermal injury. Gastrointest Endosc 56(1): 95-99.

8 Wen W, Shi C, Shi Y (2012) A pilot animal and clinical study of autologous blood solution compared with normal saline for use as an endoscopic submucosal cushion. Exp Ther Med 4(3): 419-424.

9 Uraoka T, Fujii T, Saito Y, Kuang I Fu, Daizo Saito, et al. (2005) Effectiveness of glycerol as a submucosal injection for EMR. Gastrointest Endosc 61(6): 736-740.

10 Saunders BP, Tsiamoulos ZP (2016) Endoscopic mucosal resection and endoscopic submucosal dissection of large colonic polyps. Nat Rev Gastroenterol Hepatol 13(8): 486-496.

$\begin{array}{ll}\text { BIOMEDICAL } & \text { Assets of Publishing with us } \\ \text { RESEARCHES } & \text { - Global archiving of articles } \\ \text { - Immediate, unrestricted online access } & \text { - Rigorous Peer Review Process } \\ & \text { - Authors Retain Copyrights }\end{array}$

